\section{Blood or plasma lactate?}

\section{A. R. W. Forrest, FRCPEdin, CChem, FRSC,} S. Morton, MSc, FIMLS and C. Lambardarios, MSc, FIMLS

Department of Clinical Chemistry, Royal Hallamshire Hospital, Sheffield S10 2JF, UK

\section{Sir}

The measurement of lactate as an aid in establishing optimal individual training schedules is now a common practice in both human and animal athletes. However, there does not seem to be a widespread appreciation amongst those who use lactate assays for this purpose that lactate concentrations in blood and plasma, at a particular time, may not be identical. The difference may be significant.

In the UK there is now a divergence in practice between those measuring lactate in patients and those measuring lactate in athletes. In clinical practice, samples for lactate are usually collected into tubes containing a fluoride-oxalate or fluoride-EDTA preservative and anticoagulant. These are then centrifuged and the supernatant plasma assayed for lactate. In the field, or in the exercise laboratory, athletes usually have a blood sample taken by finger prick which is assayed immediately using a small benchtop lactate analyser, such as those manufactured by Yellow Springs Instruments or Analox.

At rest, there is little difference between the blood and plasma lactate concentration. However, this is not the case after exercise. Figure 1 shows the blood and plasma lactate concentrations found in four athletes completing a half marathon. It will be seen that, whilst there is about a 10 per cent difference between blood and plasma lactate concentrations before the race, the difference rises to over 30 per cent in samples taken immediately after the finish of the race.

The relationship between plasma and blood lactate may be described by the following equation:

Blood Lac $=$ Red Cell Lac $\times$ HCT $+((1-$ HCT $) \times$ Plasma Lac $)$

\section{Lac $=$ Lactate. $\mathrm{HCT}=$ Haematocrit}

Thus, as the haematocrit rises during prolonged exercise, when some dehydration is common, the difference between the blood and plasma lactate will increase. In the present example the mean haematocrit of the four subjects at the start of the event was 0.43 rising to 0.48 by the finish.

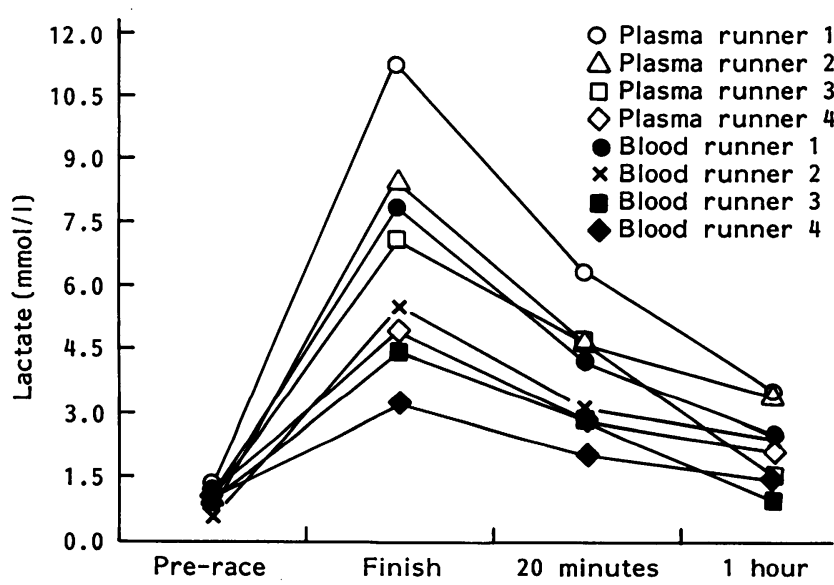

Figure 1. Blood and plasma lactate concentration in four athletes before and after completing a half marathon. Runners 1 and 3 are elite athletes, runners 2 and 4 complete at club level

In equine athletes, where there is a dramatic increase in haematocrit during exercise, produced by the release of red cells sequestered in the spleen, the difference between plasma and blood lactate during and immediately after exercise would be even more pronounced.

In general one might expect the plasma lactate to be a better guide to the metabolic state of the muscle generating lactate than a measurement of whole blood lactate. Apart from the effect of the increase in haematocrit associated with prolonged exercise described above, one would also expect there to be a blunting in the apparent blood lactate response when samples are obtained immediately after burst exercise. Under such circumstances, it will take a significant length of time for lactate to diffuse from plasma into red cells after it is generated in muscle tissue and, until equilibration has been reached, plasma lactate concentration will be a better indicator of the metabolic state of the muscle than the blood lactate.

We suggest that further work needs to be done in determining the significance of the relationship between blood and plasma lactate during and after exercise. Whether or not the theoretical advantages of measuring plasma lactate concentrations outweigh the practical convenience of measuring whole blood lactate with the instruments now available remain to be established. 